\title{
Pancreatic Lymphoma from Head to Tail
}

Bohyun Kim¹, Jei Hee Lee ${ }^{1}$, So Hyun Park², Jai Keun Kim

1 Department of Radiology, Ajou University Hospital, Suwon, South korea ${ }^{2}$ Department of Radiology, Gacheon Gil Hospital, Incheon, South Korea

Background

Pancreatic lymphoma is a rare disease that arises primarily from the pancreas or secondarily involves the pancreas as a part of systemic lymphoma. Due to its rarity, radiologic features of the disease is relatively unfamiliar with the radiologists which could potentially lead to curative-intent, yet futile surgical resection.

Clinical features of pancreatic lymphoma

\begin{tabular}{|c|c|c|}
\hline & $1^{\circ}$ Pancreatic lymphoma & $2^{\circ}$ Pancreatic lymphoma \\
\hline Prevalence & Rare; $<2 \%$ of extranodal lymphomas & Not uncommon; > 30\% in NHL \\
\hline Epidemiology & $M: F=7: 1$, seventh decade & \\
\hline Histologic subtype & Most commonly DLBCL & \\
\hline Lesion & Bulk of disease in pancreas/peripancreatic area & $\begin{array}{l}\text { Frequently as direct extension from peripancreatic } \\
\text { lymphadenopathy }\end{array}$ \\
\hline Symptoms & \multicolumn{2}{|c|}{ Abdominal pain, weight loss; infrequently jaundice } \\
\hline Lab findings & \multicolumn{2}{|c|}{ Elevated LDH, normal to slightly elevated CA 19-9 } \\
\hline Diagnosis & \multicolumn{2}{|c|}{ EUS-FNA with flow cytometry; percutaneous or open biopsy in undiagnostic cases } \\
\hline Treatment & \multicolumn{2}{|c|}{ Chemotherapy in the mainstay of the treatment (CHOP, R-CHOP) } \\
\hline Prognosis & CR/PR in $85 \%$; surveillance CT warranted & Depends on the extent of systemic disease \\
\hline
\end{tabular}

Radiologic features of pancreatic lymphoma

- Ultrasonography

- A bulky homogeneous hypoechoic mass
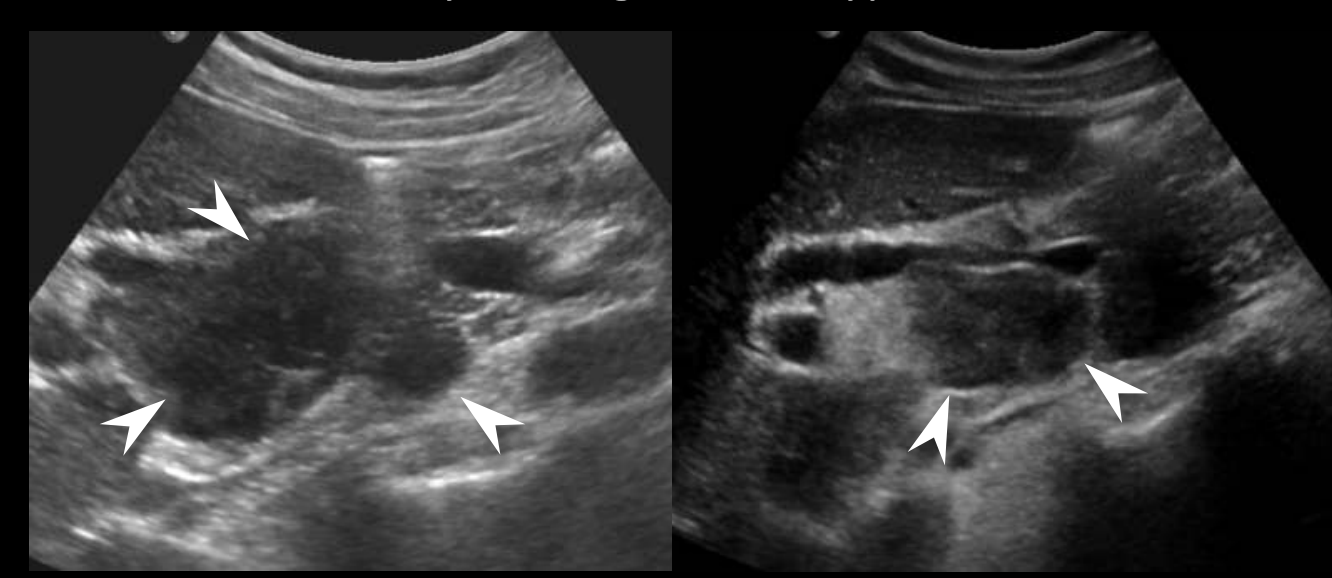

- $\mathrm{MR}$

- T1WI: Homogeneous hypo-signal intensity

- T2WI: Homogeneous hyper-signal intensity

- Mild homogeneous enhancement, but without delayed enhancement

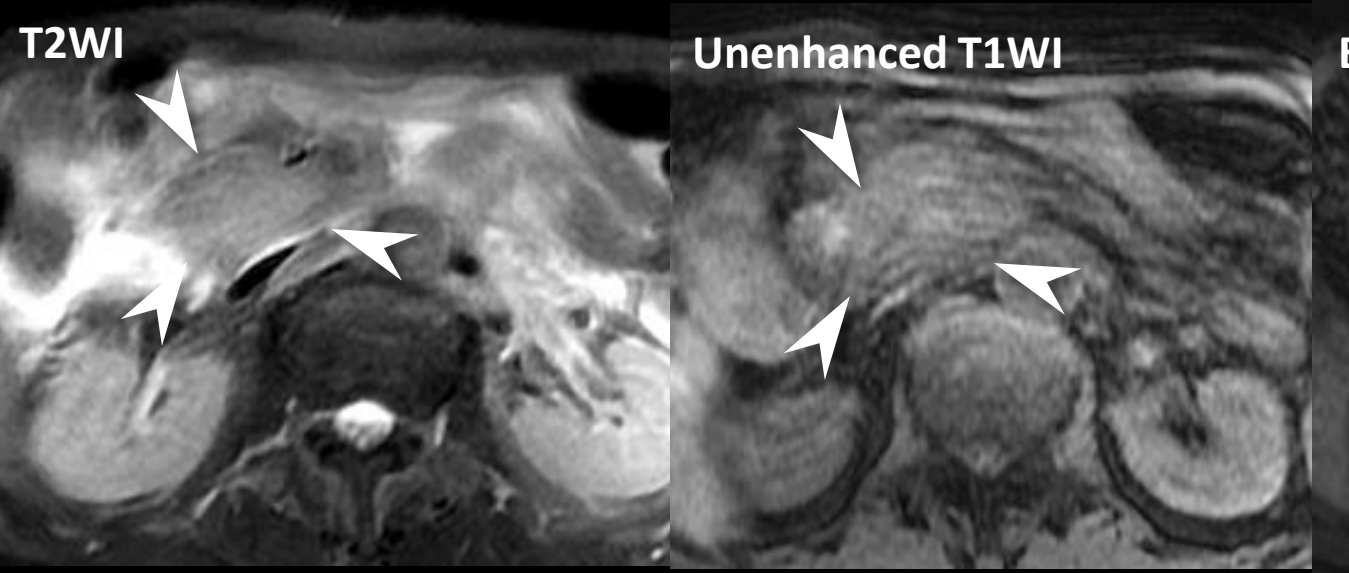

Radiologic differential diagnosis

\begin{tabular}{l} 
Pitfalls \\
\hline - Share major radiologic features \\
- Both manifests as focal lesion(s) or \\
diffuse disease
\end{tabular}

- Pancreatic cancer is the most common pancreatic neoplasm

Pancreatic cancer

Pancreatic metastasis

- Hypovascular metastasis may mimic focal lymphoma

- Hypovascular tumor (usually neuroendocrine carcinoma)

- Well-defined, less likely to cause pancreatic duct obstruction
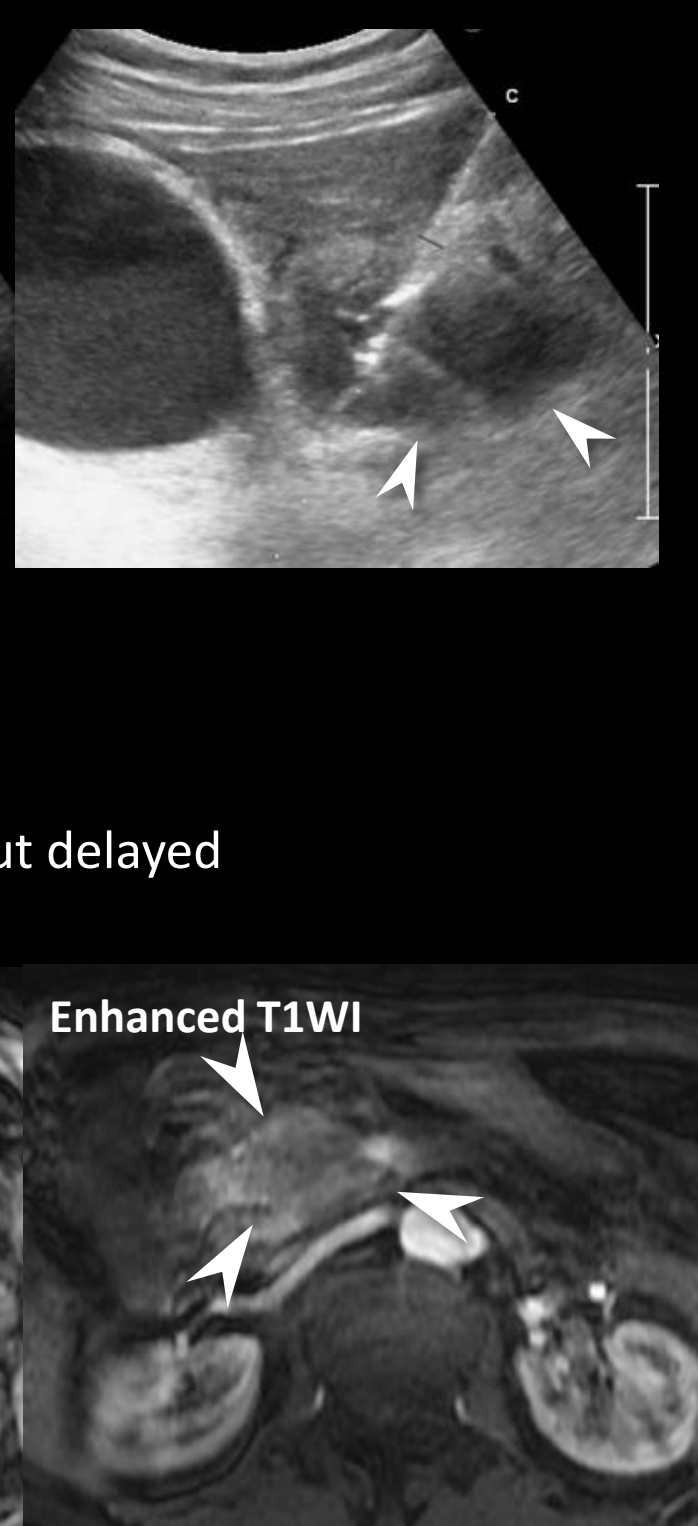

- $\mathrm{CT}$

- Large, well-defined homogeneously hypovascular mass(es)

- Without pancreatic duct obstruction/vascular invasion

- Two morphologic patterns: Localized mass(es) and diffuse disease
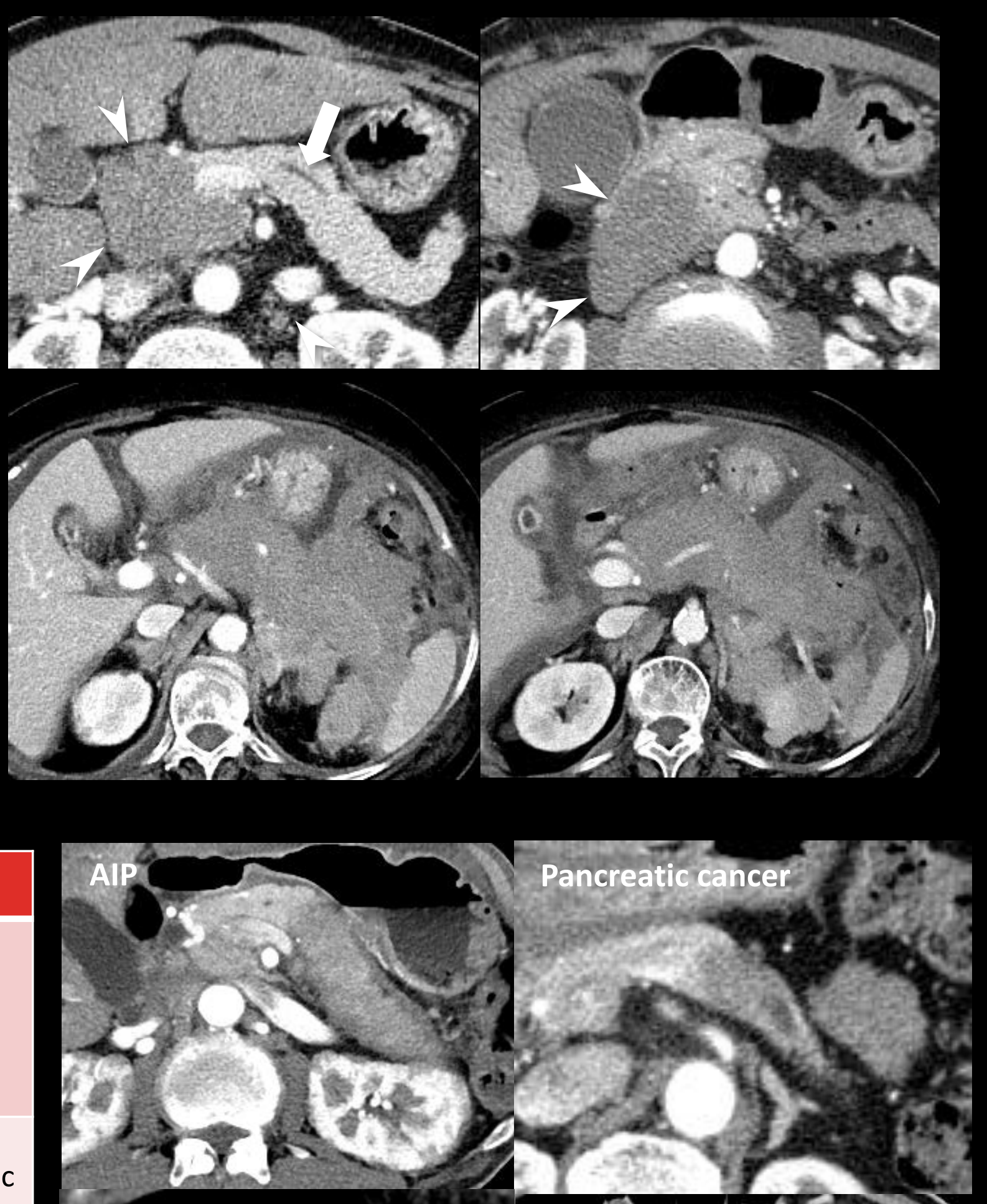

- Progressive enhancement and peripancreatic infiltration causing ductal obstruction

- History of known primary cancer

- Hypervascularity favors metastasis

- Internal necrosis and occasional calcification

- Distant metastasis

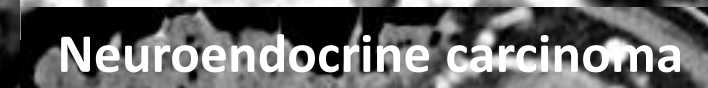

Pancreatic metastasis

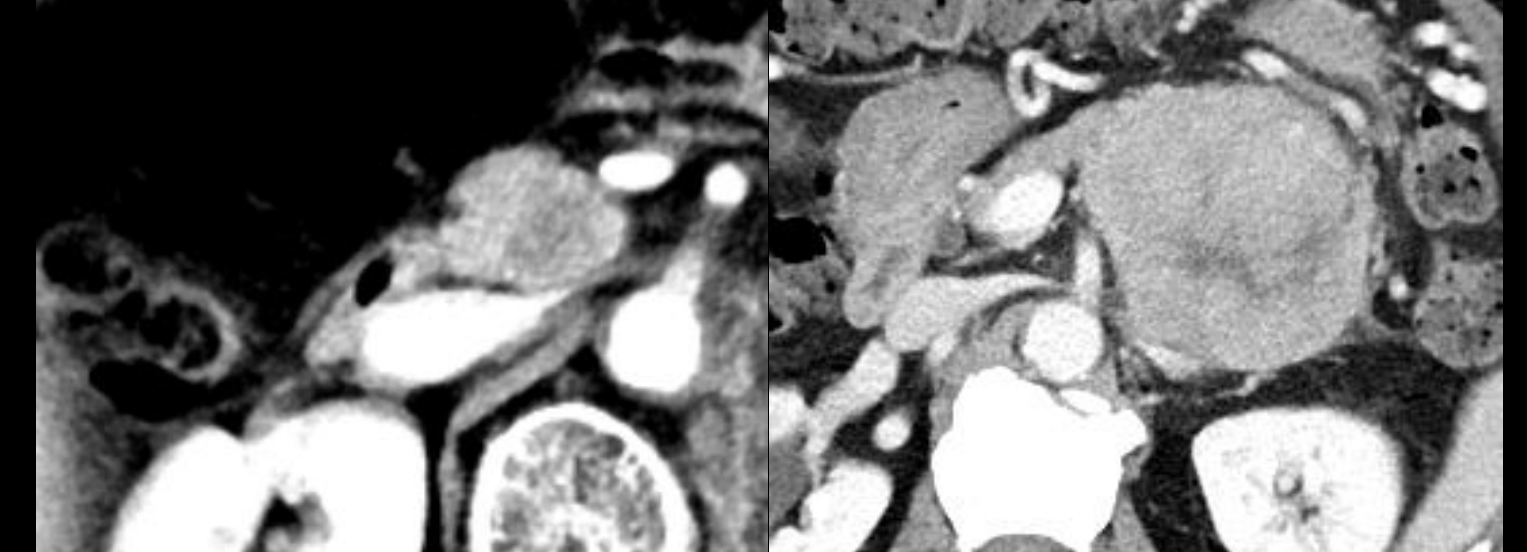

